\title{
Ultraviolet Laser Based Gas Phase Fluorination of Polystyrene
}

\author{
Simon Kibben ${ }^{*}$, Klaus Vogelsang ${ }^{2}$ \\ ${ }^{1}$ BIAS-Bremer Institut für angewandte Strahltechnik GmbH, Bremen, Germany \\ ${ }^{2}$ INNOVENT e.V., Jena, Germany \\ Email: ${ }^{*}$ kibben@bias.de
}

Received 19 June 2014; revised 27 July 2014; accepted 20 August 2014

Copyright (C) 2014 by authors and Scientific Research Publishing Inc.

This work is licensed under the Creative Commons Attribution International License (CC BY).

http://creativecommons.org/licenses/by/4.0/

c. (i) Open Access

\begin{abstract}
A novel hybrid process for surface fluorination of polymers is being introduced. The process is based on ultra violet (UV) laser radiation, which on the one hand forms radicals out of an atmosphere of a partially fluorinated benzene, and on the other hand activates a polymer surface in the areas where the UV radiation hits the surface. The radicals can react with the polymer surface, hence altering the surface energy. With this process, a fluorine content of over $30 \%$ on the surface of bulk polystyrene can be achieved, while the smallest possible structure size was smaller than 1 mm.
\end{abstract}

\section{Keywords}

UV-Laser Polymer Modification, Local Fluorination

\section{Introduction}

The process of direct fluorination is a well-known tool to enhance the characteristics of mainly polymer surfaces. Friedrich et al. showed that by adding fluorine into polymeric surfaces, out gassing of hydrocarbons can be inhibited [1]. Also, the wettability of the surface can be altered as the surface energy changes with the introduction of fluorine [2].

Usually, direct fluorination is done utilizing gaseous fluorine in a sealed chamber. The process of direct fluorination does not allow to fluorinate a surface only locally. To achieve a local fluorination, several process steps have to be added: A sacrificial layer is placed in the areas, where fluorine must not react with the surface. Also, plasma fluorination processes do exist, where a plasma containing fluorine radicals is applied to the surface locally. Usually these plasmas have a Gaussian distribution of the radicals in the plasma flame and smallest struc-

\footnotetext{
${ }^{*}$ Corresponding author.
}

How to cite this paper: Kibben, S. and Vogelsang, K. (2014) Ultraviolet Laser Based Gas Phase Fluorination of Polystyrene. Journal of Surface Engineered Materials and Advanced Technology, 4, 309-317. 
tures achievable are in the range of $10 \mathrm{~mm}$ [3].

Ikegame and Murahara introduced a process, in which the fluorine is only present in a bonded state $\left(\mathrm{CF}_{4}\right)$, thus not reacting with the surface. The fluorine bonds of the molecule then are being cracked by UV photons emitted by a $\mathrm{Xe}_{2}{ }^{*}$ excimer lamp [4]. The C-H bonds of the polymer surface are activated by an ArF excimer laser to dissociate the $\mathrm{C}-\mathrm{H}$ bonds. The hydrogen reacts with the fluorine radical, and the $\mathrm{CF}_{3}$ molecules bond to the dangling bond of the polymer. In combination, the fluorine radical easily can react with the areas of the polymer surface, where the excimer laser photons activated the polymer bonds. This method offers the possibility to fluorinate a polymeric surface without the use of the highly toxic gaseous fluorine. The process also was adapted for a local fluorination. This was achieved in a two-step process. First the whole surface was fluorinated by putting a drop of perfluoropolyether onto the polymer surface and covering it with a fused silica window. This was then irradiated with a $\mathrm{Xe}_{2}$ * excimer lamp, allowing the hydrogen to be replaced with $\mathrm{F}$ and $\mathrm{CF}_{3}$. In the second step, water was put onto the surface and was covered with a fused silica window. This then was irradiated with an ArF excimer laser through a reticle to locally modify the polymer surface [5] by the addition of $-\mathrm{OH}$ groups.

Here we suggest a novel hybrid process, where a fluorine containing precursor and a polymer surface is activated by one and the same UV laser pulse. Thus, the fluorine only is generated in an area where it should react with the polymeric surface. With this process it is possible to introduce the fluorine only in small confined areas, thus providing a one-step process for local fluorination.

\section{Theoretical Background}

In order to allow the local fluorination to take place using one UV source, several requirements have to be fulfilled. The polymer and the precursor need to be activated by the same photons, thus absorb the same photons. Only then the precursor releases fluorine radicals and the polymer gets activated to accept the radicals easily.

The density of the precursor molecules is also crucial. If the density is low, only few radicals will be formed. The mean free path of the radicals will be high and the diffusion of the radicals will then lead them far away from the area where the UV radiation took effect. This generates a slow decay of fluorine at the edges of this area. Even without UV activation of the polymer, the radicals can attach themselves to the polymer chain.

When the density of the precursor molecules is too high, the fluence of the UV radiation will strongly decay. Only few UV photons will reach the polymer surface and most of the fluorine radicals are generated far away from the polymer surface.

A physical model describing the processes of absorption, radical formation, radical diffusion and the pathways of the radical to the polymer surface and its results has been published elsewhere [6]. The model was adapted to a precursor, which is being ionized by absorbing two photons, as described in [7]. This leads to a disproportional dependance of the radical density to the pulse fluence or the photon flux, respectively.

\section{Experimental}

\subsection{Materials}

As polymer, polystyrene (PS) was considered which was manufactured by Rocholl GmbH, Germany, in plates of $148 \mathrm{~mm} \times 210 \mathrm{~mm}$ with a thickness of $3 \mathrm{~mm}$. The average peak-to-valley height of the surface was $30 \mathrm{~nm}$. It is known that PS has a strong absorption in the UV range [8]. The material was cut into samples with a milling machine and cooled during this process with deionized water. The samples were cleaned with isopropyl alcohol for two minutes in an ultrasonic bath.

1,2,3,5-Tetrafluorobenzene (CAS 23367-82-0) was selected as a fluorine containing precursor. The precursor was delivered by abcr GmbH, Germany, and had a purity of $97 \%$ and was used as delivered. The precursor is ionizable by absorption of two photons with an energy of 5 electron volts in a time of a few nanoseconds [7].

\subsection{Methods}

As UV source, an excimer laser Lambda Physik LPX 305i was employed. The laser had a krypton fluorine gas filling (emission wavelength: $248 \mathrm{~nm}$ ). The pulse energy could be adjusted via a motorized attenuator. The original dimensions of the beam were $12 \mathrm{~mm} \times 25 \mathrm{~mm}$, the beam was expanded to a diameter of $40 \mathrm{~mm} \times 50 \mathrm{~mm}$ using four cylindrical lenses. The beam could partially be blocked with a shadow mask, three stripes of $5 \mathrm{~mm}$ 
width and a pitch of $10 \mathrm{~mm}$ were then irradiated. Also, a slit with a width of $0.5 \mathrm{~mm}$ or a second shadow mask shown in Figure 7(b) could be placed in the beam. The slit (or the mask) was imaged onto the sample surface using a $50 \mathrm{~mm}$ diameter biconvex lens with a focal length of $150 \mathrm{~mm}$. Parameters which were kept constant for all experiments are mentioned in Table 1. For each sample, a pulse fluence and an accumulated fluence were specified. The pulse fluence was adjusted and kept constant during the irradiation using the motorized attenuator, the accumulated fluence is defined by the number of pulses.

The laser radiation was directed into a vacuum chamber which could be flushed with nitrogen and the precursor. The chamber had a calcium fluoride window allowing the radiation to enter the chamber.

Each sample was placed in a distance of $1 \mathrm{~mm}$ to the window. The chamber was evacuated to $1 \mathrm{mbar}$ and flushed with nitrogen for two times prior to filling the chamber with the precursor.

Three types of experiments were carried out. In the first type of experiment, nine samples were irradiated homogeneously over the sample surface with different pulse fluences and accumulated fluences. The parameters are given in Table 2. In the results section, these samples will be referred to as sample type H-XX-YY where $\mathrm{H}$ stands for homogeneous, with $\mathrm{XX}$ being the pulse fluence in $\mathrm{mJ} / \mathrm{cm}^{2}$ and $\mathrm{YY}$ being the accumulated fluence in $\mathrm{J} / \mathrm{cm}^{2}$.

In the second experiment, two samples were irradiated through the shadow mask with a slit width of $5 \mathrm{~mm}$, only the accumulated fluence was varied (see Table 3 for parameters). These samples will be named M-XX, where M stands for Mask with XX being the accumulated fluence of the sample in $\mathrm{J} / \mathrm{cm}^{2}$. In the third experiment, three samples were irradiated stepwise through the slit aperture of $0.5 \mathrm{~mm}$ width, which was imaged onto the sample surface. On each sample the slit was placed in three different positions ( $2 \mathrm{~mm}$ distance to each other) and the sample was irradiated with a different accumulated fluence for each slit position. For each sample, a different precursor density/pressure was chosen (see Table 4 for parameters). The samples will be named S-XX where $\mathrm{S}$ stands for slit with XX being the precursor pressure in mbar.

One further sample was prepared for demonstrating the effect of local fluorination. The surface of this sample was grinded manually with a 500 grit sandpaper under running water. The sample again was cleaned with isopropyl alcohol for two minutes in an ultrasonic bath. A shadow mask (depicted in Figure 7(b)) was placed at the same position of the $0.5 \mathrm{~mm}$ slit. Thus, the mask was imaged onto the surface. The sample then was irradiated with the excimer laser with a pulse fluence of $10 \mathrm{~mJ} / \mathrm{cm}^{2}$ and an accumulated fluence of $30 \mathrm{~J} / \mathrm{cm}^{2}$, the precursor pressure was set to 65 mbar.

All samples (except for the demonstration sample) were analyzed using X-ray photoelectron spectroscopy (XPS). The measurement results of all XPS measurements are amount-of substance-fractions for all elements except for hydrogen. Due to the method itself, hydrogen is not detectable. The measurement spot of the XPS was $500 \mu \mathrm{m}$ for the homogeneously irradiated samples (one spot) and the samples structured with the $5 \mathrm{~mm}$ shadow mask (line scan) and $100 \mu \mathrm{m}$ for the samples structured with the $0.5 \mathrm{~mm}$ slit aperture (line scan). As a reference, an untreated sample (only cleaned as described above) and one sample which was directly fluorinated (Parameters: 40 mbar fluorine, 360 mbar nitrogen, duration: $180 \mathrm{~s}$ ) were analyzed using XPS and attenuated total reflectance fourier transform infrared spectroscopy (ATR-FTIR). The surface of a sample of series S was investigated using scanning electron microscopy (SEM). Contact angle measurements with ethylene glycol, thiodiglycol and diiodomethane were performed on samples of series $\mathrm{H}$. The results were used to calculate the polar and dispersive component of the surface energy.

\section{Results}

An untreated sample (only cleaned in isopropyl alcohol) was analyzed as a reference using XPS. The sample consisted of $0.6 \%$ oxygen, the rest was carbon. The directly fluorinated sample consisted of $40.8 \%$ fluorine, $2.7 \%$ oxygen, the rest was carbon. It is not known if the oxygen is present as a residual gas inside the free volume of the polymer, as an oxygen-containing contaminant or if it is bonded to the polymer.

The measured ATR-FTIR spectra of both samples did not show any significant differences of both samples in the C-F band $\left(1400 \mathrm{~cm}^{-1}\right.$ to $\left.1000 \mathrm{~cm}^{-1}\right)$ or in any other region. Presumably the modified layer is only a few nanometers deep and thus does not significantly influence the ATR-FTIR spectra.

In Figure 1(a) and Figure 1(b), the fluorine content of all samples of series $\mathrm{H}$ is shown over the processing time. In Figure 2(a), the fluorine content of homogeneously irradiated samples over the accumulated fluence is depicted. The oxygen content of all samples of series $\mathrm{H}$ is depicted in Figure 2(b). The fluorine content of the 
Table 1. Constant parameters of sample irradiation.

\begin{tabular}{llll}
\hline Parameter & Value & Unit \\
\hline Laser wavelength & 248 & $\mathrm{~nm}$ & ${ }^{\circ} \mathrm{C}$ \\
Temperature & 23 & $\mathrm{~Hz}$ & \\
Repetition rate & 50 & $\mathrm{~Hz}$ \\
\hline
\end{tabular}

Table 2. Parameters of homogeneous sample irradiation (series H).

\begin{tabular}{lll}
\hline Parameter & Value & Unit \\
\hline Pulse fluence & $1 ; 10 ; 25 ; 50$ & $\mathrm{~mJ} / \mathrm{cm}^{2}$ \\
Accumulated fluence & $30 ; 60 ; 90$ & $\mathrm{~J} / \mathrm{cm}^{2}$ \\
Precursor pressure & 11 & $\mathrm{mbar}$ \\
\hline
\end{tabular}

Table 3. Parameters of shadow mask sample irradiation (sample M).

\begin{tabular}{lll}
\hline Parameter & Value & Unit \\
\hline Pulse fluence & 1 & $\mathrm{~mJ}^{\prime} \mathrm{cm}^{2}$ \\
Accumulated fluence & 60 & $\mathrm{~J} / \mathrm{cm}^{2}$ \\
Precursor pressure & 11 & $\mathrm{mbar}$ \\
Aperture width & 5 & $\mathrm{~mm}$ \\
\hline
\end{tabular}

Table 4. Parameters of slit aperture sample irradiation (series S).

\begin{tabular}{lll}
\hline Parameter & Value & Unit \\
\hline Pulse fluence & 10 & $\mathrm{~mJ} / \mathrm{cm}^{2}$ \\
Accumulated fluence & $30 ; 60 ; 90$ & $\mathrm{~J} / \mathrm{cm}^{2}$ \\
Precursor pressure & $1 ; 11 ; 65$ & $\mathrm{mbar}$ \\
Aperture width & 0.5 & $\mathrm{~mm}$ \\
\hline
\end{tabular}

a)

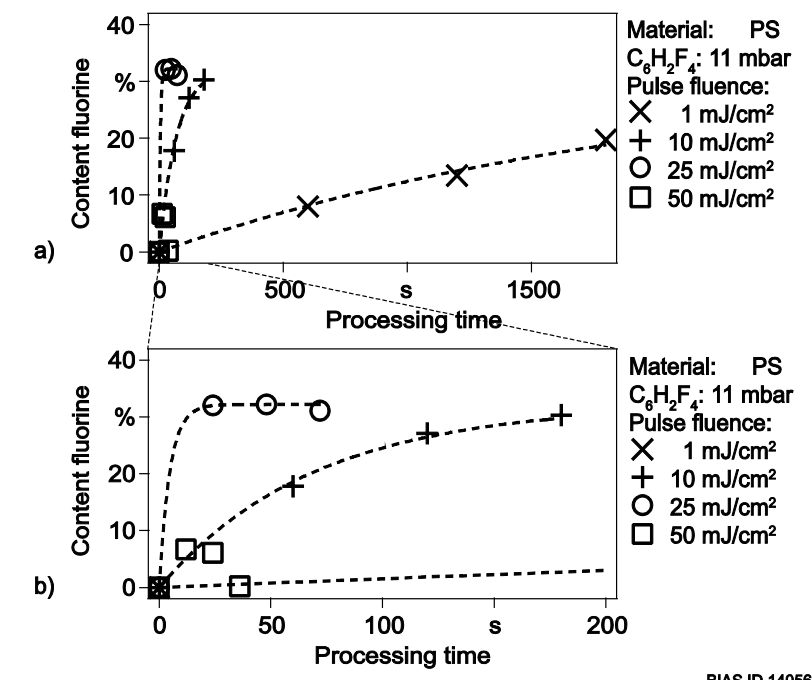

Material: PS

$\mathrm{C}_{8} \mathrm{H}_{2} \mathrm{~F}_{4}: 11$ mbar

Pulse fluence:

X $1 \mathrm{~mJ} / \mathrm{cm}^{2}$

$+10 \mathrm{~mJ} / \mathrm{cm}^{2}$

O $25 \mathrm{~mJ} / \mathrm{cm}^{2}$

$\square 50 \mathrm{~mJ} / \mathrm{cm}^{2}$

Figure 1. Measured content of fluorine over the processing time for different pulse fluences of the samples of series $\mathrm{H}$. In a), the processing time from $0 \mathrm{~s}$ to $1800 \mathrm{~s}$ is depicted; b) is a detailed view of the first $200 \mathrm{~s}$ of the processing time.

homogeneously irradiated sample series $\mathrm{H}$ not only depends on the accumulated fluence, but also on the pulse fluence. The fluorine content of the samples with a pulse fluence of $1 \mathrm{~mJ} / \mathrm{cm}^{2}$ reaches $19.7 \%$ at an accumulated fluence of $90 \mathrm{~J} / \mathrm{cm}^{2}$, while the samples irradiated with a pulse fluence of $25 \mathrm{~mJ} / \mathrm{cm}^{2}$ reaches a fluorine content of 


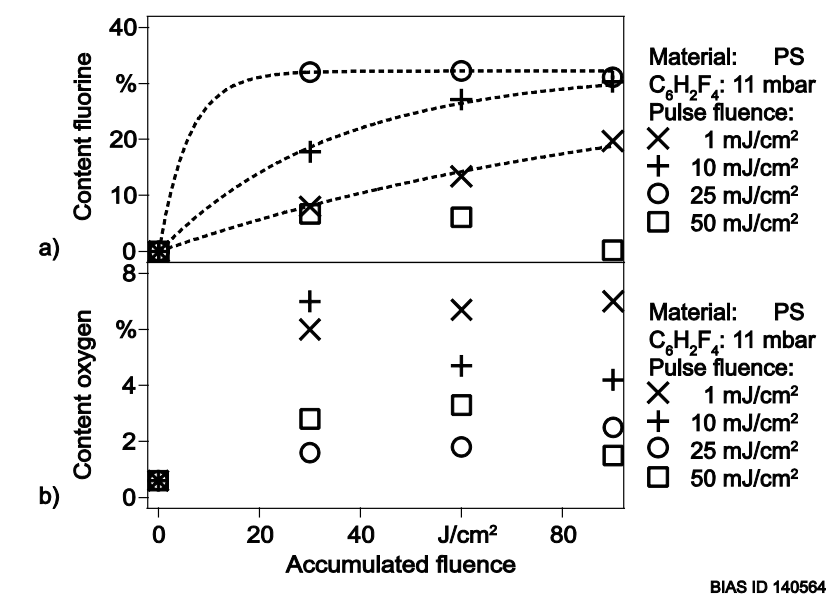

Figure 2. Content of a) fluorine and b) oxygen over the accumulated fluence for different pulse fluences.

$32 \%$ at an accumulated fluence of $30 \mathrm{~J} / \mathrm{cm}^{2}$. For pulse fluences of 1,10 and $25 \mathrm{~mJ} / \mathrm{cm}^{2}$, the fluorine content in-creases exponentially according to a first order chemical reaction. For a pulse fluence of $50 \mathrm{~mJ} / \mathrm{cm}^{2}$, the fluorine content does not increase exponentially, but has its maximum of $6.7 \%$ at an accumulated fluence of $30 \mathrm{~J} / \mathrm{cm}^{2}$ and decreases with higher accumulated fluences. The trend lines in Figure 1(a) and Figure 1(b) and Figure 2(a) are according to a chemical reaction model explained in chapter 5 . The oxygen content of all samples of series $\mathrm{H}$ is strongly increased compared to an untreated sample. The oxygen content of all samples of the series $\mathrm{H}$ lies between 1.5\% (H-50-90) and 7\% (H-10-30 and H-01-90). No clear tendency of the oxygen content over pulse and accumulated fluence can be pointed out.

The results of a XPS line scan of sample M is shown in Figure 3. The sample shows a fluorine content of up to $14.8 \%$ in the locally fluorinated areas. In the unirradiated areas the fluorine content mostly is lower than the measurement threshold $(0.2 \%)$. The edges between these areas are sharp. The oxygen content varies between $9.9 \%$ and $14.8 \%$ but mainly is at about $11 \%$. The irradiation does not seem to have an influence on the oxygen content.

The fluorine content of all samples of type $\mathrm{S}$ shows a broadening of the irradiated areas. The fluorine and oxygen content of the samples is shown in Figures 4(a)-(c). The fluorine content also is quite high in the areas close to the irradiation and the edges are partially very broad. The sample S-01 has an increased fluorine content in the irradiated areas with increasing accumulated fluence (see Figure 4(a)). The left peak was irradiated with $30 \mathrm{~J} / \mathrm{cm}^{2}$, the middle peak was irradiated with $60 \mathrm{~J} / \mathrm{cm}^{2}$ and the right one was irradiated with $90 \mathrm{~J} / \mathrm{cm}^{2}$. This is the case for all samples. The increase is not that strong for the sample S-11 (see Figure 4(b)). At sample S-65, there is no significant difference in fluorine concentration at the maxima at all, but the broadening of the irradiated areas is much smaller for the region irradiated with $30 \mathrm{~J} / \mathrm{cm}^{2}$ than for the regions irradiated with $60 \mathrm{~J} / \mathrm{cm}^{2}$ or $90 \mathrm{~J} / \mathrm{cm}^{2}$ (see Figure $4(\mathrm{c})$ ).

At the sample S-01, the measured peaks have a shape, which can be described by the sum of two Gaussian functions, both having different widths. The peaks of sample S-11 also look like having two regions with different slopes, one steep close to the irradiated areas with high fluorine content and a more shallow one further away from the irradiated areas with lower fluorine content. At sample S-65, each irradiated area has a different slope, while the slope is the steepest for the smallest accumulated fluence $\left(30 \mathrm{~J} / \mathrm{cm}^{2}\right)$ and gets lower for higher fluences.

The surface of the sample S-65 was inspected using scanning electron microscopy (SEM). The results are shown in Figure 5. An image of the whole surface as well as a magnification of an edge between an untreated and an irradiated area was taken. Both areas were analyzed using secondary electrons (SE) and backscattered electrons (BSE). SE is used to show irregularities of the sample topography. With BSE, differences in sample composition can be detected. The surface of the sample does not show any roughening or other effects which could be due to the local fluorination process in the SE images. In the BSE images, a difference between the untreated and UV-fluorinated areas is clearly noticeable. The locally fluorinated areas have relatively sharp edges.

In Figure 6 the measured surface energy of homogeneously UV-fluorinated samples of series $\mathrm{H}$ is shown. 

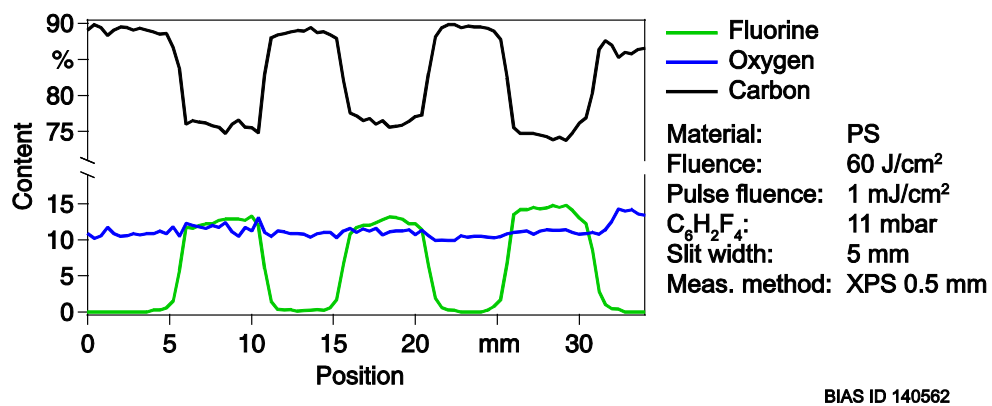

Figure 3. Content of fluorine, oxygen and carbon of sample M over the position on the sample.

a)
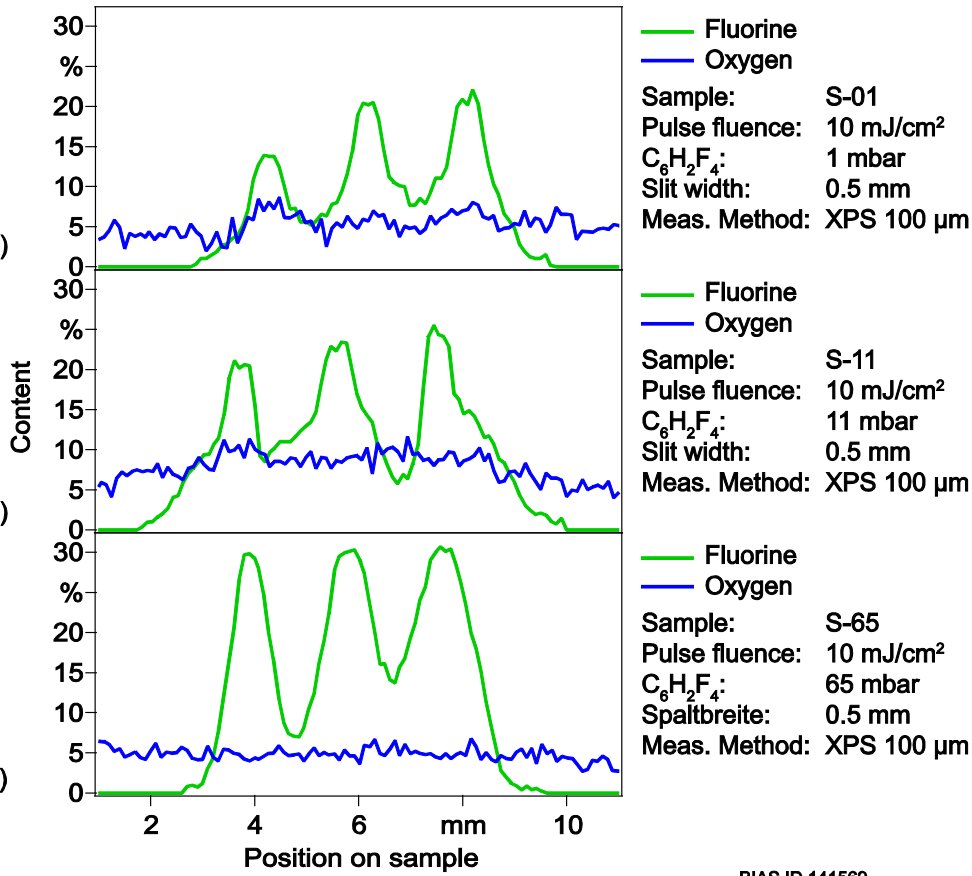

Figure 4. Content of fluorine and oxygen of the samples of series S over the position on the sample.

The surface energy decreases with increased accumulated fluence. Although the overall surface energy decreases, the polar component of the surface energy increases.

In Figure 7(a), a photograph of the demonstration sample is shown after it was taken out of deionized water. The uniformly distributed water droplets are clearly visible on the sample surface. The diameter of the droplets varies between $0.32 \mathrm{~mm}$ and $0.77 \mathrm{~mm}$, the mean diameter is $0.50 \mathrm{~mm}$. With an estimated height of the droplets of $0.2 \mathrm{~mm}$, the volume of each droplet is $0.02 \mu \mathrm{l}$.

\section{Discussion}

All homogeneously irradiated samples have a remarkably high fluorine content of up to 32.2\%. The fluorine content depends on the accumulated fluence and the pulse fluence. The fluorination reaction of the polymer surface can be expressed by a first-order chemical reaction: $c_{F}=F_{\max } \cdot\left(1-e^{-k_{R_{a d}}^{t}}\right) . \quad C_{F}$ is the fluorine content on the polymer surface, $F_{\max }$ is the maximum fluorine concentration which can be achieved, $\mathrm{k}$ is the reaction constant, $\mathrm{n}_{\mathrm{Rad}}$ is the radical density on the polymer surface at the (temporal) end of a laser pulse and $\mathrm{t}$ is the time of the overall process. The $\mathrm{n}_{\text {Rad }}$ can be obtained with the model described in [6]. The ionization process de- 
a)
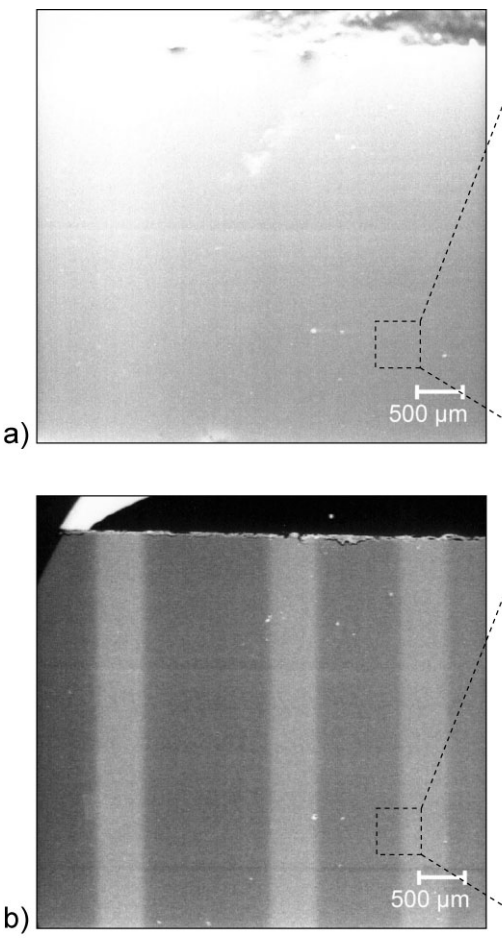
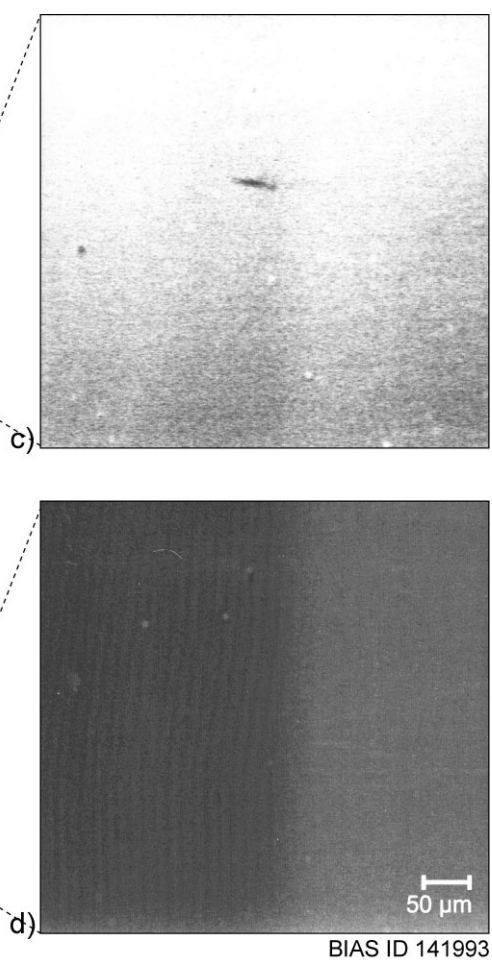

Figure 5. SEM images of the sample S-65. In a), the surface of the sample in SE mode is shown; in b), the same area is shown in BSE mode. The three white stripes are the locally fluorinated areas (right: $30 \mathrm{~J} / \mathrm{cm}^{2}$, middle: $60 \mathrm{~J} / \mathrm{cm}^{2}$, left: $90 \mathrm{~J} / \mathrm{cm}^{2}$ ). c) and d) are details of a) and b), respectively.

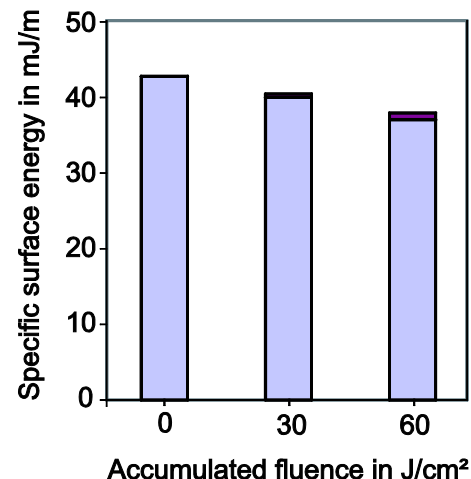

-Polar component 口Dispersive component

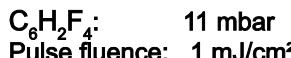

Pulse fluence: $1 \mathrm{~mJ} / \mathrm{cm}^{2}$

BIAS ID 141994

Figure 6. Measured surface energy of homogeneously UV-fluorinated samples of series $\mathrm{H}$.

scribed in the paper has to be adapted to a two-stage photon absorption including an intermediate energy level. This adaption may be taken from [7]. The experimental parameters of the sample series $\mathrm{H}$ are used to calculate the expected fluorine content. The expected fluorine content over time or accumulated fluence is depicted as trend lines in Figure 1(a), Figure 1(b) and Figure 2(a).

The fluorine content of the edges decreases rapidly outside the irradiated areas, especially of sample M-60. On the samples of series $\mathrm{S}$, the fluorine content does not decrease that rapidly. The different behavior might be due to several reasons. First, the possible chemical reactions between the precursor and the precursor are examined.

The precursor itself is dissociated via two absorbed UV-photons, resulting in two radicals, a small one consisting of a single atom (fluorine or hydrogen) and a large one, which is the rest of the precursor $\left(\mathrm{C}_{6} \mathrm{H}_{2} \mathrm{~F}_{3} \cdot\right.$ or 
$\mathrm{C}_{6} \mathrm{HF}_{4}$ ). The radical can react with another single atom radical, a precursor molecule, a dissociated precursor molecule radical and the polymer (which is the intended reaction). The dissociated precursor radical has the same possible reaction partners.

Depending on the pulse fluence, the dissociated precursor molecule density and thereby the radical density is about three orders of magnitude below the precursor density of non-dissociated molecules. Hence the probability of a reaction between two radicals is very low compared to reactions between the dissociation products and a precursor molecule. These reactions are estimated to have only low influence on the process.

Most of the ongoing reactions there for will be between the precursor or the polymer surface and a dissociation product of the precursor. The suggested main reactions are depicted in Figure 8. The probability to react with the surface depends on the initial distance between the dissociation product and the polymer surface. This is extensively discussed in [6].

When a single atom radical or the dissociated precursor radical reacts with the polymer or the precursor molecule, a polymer radical and further molecules (besides photo-dissociation products and precursor molecules) might be generated. The polymer radical may be formed at the aliphatic polymer chain or at the aromatic side groups. The position of the radical depends on steric, energetic and kinetic aspects. Especially if a fluorine radical reacts with the polymer, hydrogen fluoride (HF) will be generated. Other reactions generating HF might be the reaction of a fluorine radical with a precursor molecule. The radical in the polymer molecule can abstract fluorine from a fluorine containing molecule, which may be a precursor molecule or hydrogen fluoride, which is the reaction that increases the fluorine content of the polymer.

\section{Conclusion}

The possibility of a novel one-step process for local fluorination of polymers was investigated. It was shown that a fluorine content of up to $32.2 \%$ of the polystyrene surface can be achieved using an krypton fluoride excimer laser and 1,2,3,5-Tetrafluorobenzene as a fluorine containing precursor. Relatively sharp edges of fluorinated

a)

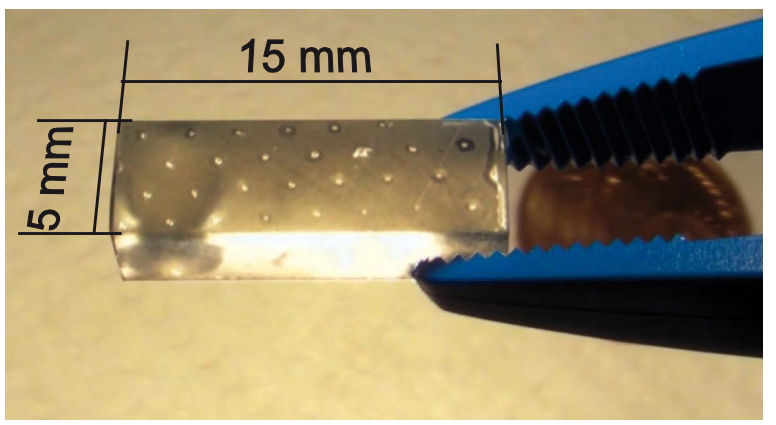

b)

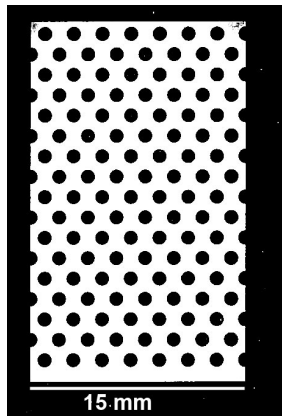

BIAS ID 141570

Figure 7. a) Locally UV-fluorinated demonstration sample with uniformly distributed water droplets on its surface. The water droplets are located in the non-fluorinated areas; b) Shadow mask used for local fluorination of the sample.

Photodissociation

$$
\begin{array}{r}
\mathrm{C}_{6} \mathrm{H}_{2} \mathrm{~F}_{4} \stackrel{+2 \mathrm{hv}}{\longrightarrow} \mathrm{C}_{6} \mathrm{HF}_{4} \cdot+\mathrm{H} \bullet \\
\mathrm{C}_{6} \mathrm{H}_{2} \mathrm{~F}_{3} \cdot+\mathrm{F} \cdot
\end{array}
$$

Reactions of fluorine Radical with the Precursor

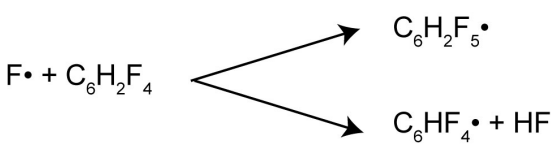

Reactions of fluorine radical with PS

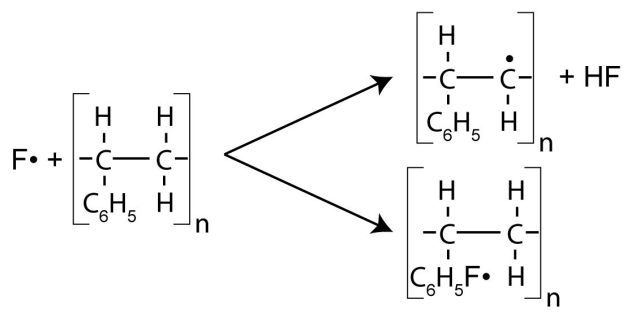


areas can be achieved by selective irradiation. The surface energy of the UV-fluorinated surface decreases with increased UV laser fluence. No surface roughening could be observed using SEM. This process offers an alternative to the conventional direct fluorination, where a local fluorination requires several process steps including masking with a sacrificial layer and removing the same after the fluorination.

\section{Acknowledgements}

The authors gratefully acknowledge the financial support of this work by the German Research Foundation under the grant VO530/50-1 and SCHI1138/1-1.

\section{References}

[1] Friedrich, J., Wigan, L., Unger, W., Lippitz, A., Erdmann, J., Gorsler, H.-V., Prescher, D. and Wittrich, H. (1995) Barrier Properties of Plasma and Chemically Fluorinated Polypropylene and Polyethylene Terephthalate. Surface and Coatings Technology, 74-75, 910-918. http://dx.doi.org/10.1016/0257-8972(95)08286-7

[2] Schonhorn, H. and Ryan, F.W. (1966) Wettability of Polyethylene Single Crystal Aggregates. The Journal of Physical Chemistry, 70, 3811-3815. http://dx.doi.org/10.1021/j100884a009

[3] Jourdain, R., Castelli, M., Shore, P., Sommer, P. and Proscia, D. (2013) Reactive Atom Plasma (RAP) Figuring Machine for Meter Class Optical Surfaces. Production Engineering, 7, 665-673. http://dx.doi.org/10.1007/s11740-013-0467-1

[4] Ikegame, T. and Murahara, M. (1998) ArF Excimer Laser and $\mathrm{Xe}_{2} *$ Excimer Lamp Induced Photochemical Fluorination of Polyimide Film. MRS Proceedings, 544, 227-232. http://dx.doi.org/10.1557/PROC-544-227

[5] Murahara, M. and Sato, Y. (2006) Photochemical Micro-Pattern Substitution of Functional Groups for Protein Attachment Control. MRS Proceedings, 950, 57-62. http://dx.doi.org/10.1557/PROC-0950-D06-04

[6] Kibben, S. (2014) Model for a UV Laser Based Local Polymer Surface Halogenation Process Using a Gaseous Precursor. Modeling and Numerical Simulation of Material Science, 4, 14-19. http://dx.doi.org/10.4236/mnsms.2014.41003

[7] Bischel, W.K., Jusinski, L.J., Spencer, M.N. and Eckstrom, D.J. (1985) Absolute Two-Photon Ionization Yields for Selected Organic Molecules at $248 \mathrm{~nm}$. Journal of the Optical Society of America B Optical Physics, 2, 877-885. http://dx.doi.org/10.1364/JOSAB.2.000877

[8] Li, T., Zhou, C. and Jiang, M. (1991) UV Absorption Spectra of Polystyrene. Polymer Bulletin, 25, 211-216. http://dx.doi.org/10.1007/BF00310794 
Scientific Research Publishing (SCIRP) is one of the largest Open Access journal publishers. It is currently publishing more than 200 open access, online, peer-reviewed journals covering a wide range of academic disciplines. SCIRP serves the worldwide academic communities and contributes to the progress and application of science with its publication.

Other selected journals from SCIRP are listed as below. Submit your manuscript to us via either submit@scirp.org or Online Submission Portal.
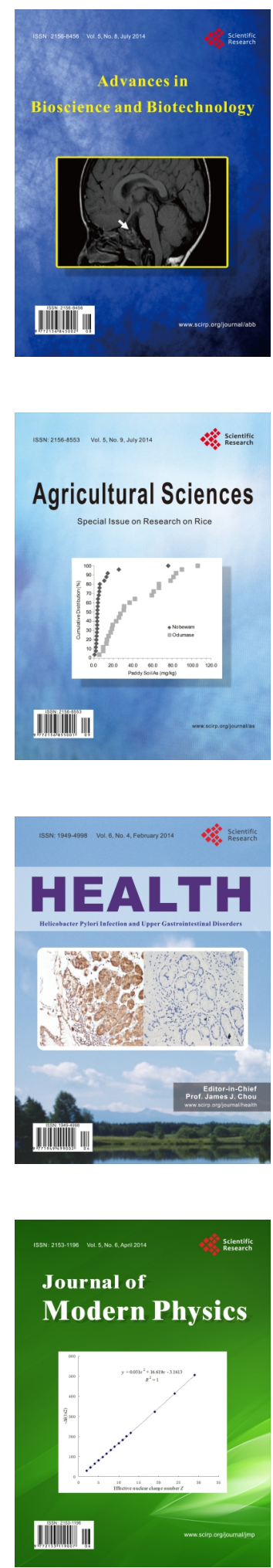
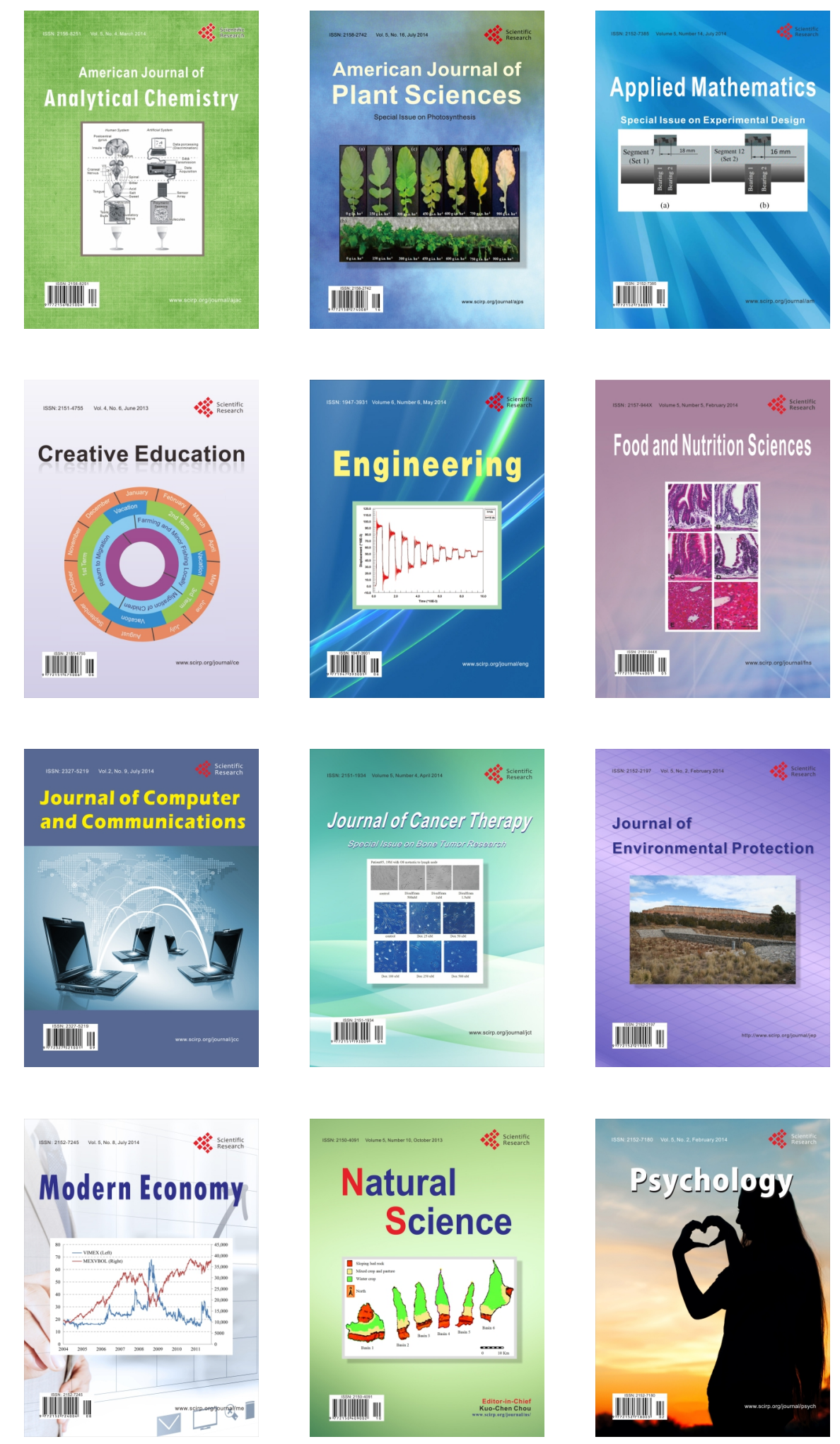